\title{
Problems of Guided Implant Surgery
}

\author{
Pravinkumar G Patil \\ International Journal of Prosthodontics and Restorative Dentistry (2020): 10.5005/jp-journals-10019-1297
}

Ideal implant placement is the ultimate goal of any clinician that can achieve high patient satisfaction with improved functional and esthetic implant restorations without complications. Conventional freehand implant surgery has been practiced by dental implant clinicians since the osseointegrated implants have been introduced. ${ }^{1}$ Computer-guided implant surgery (GIS) was first introduced in the early 2000 s to practice ideal implant placement. ${ }^{2}$ The GIS involves a reverse engineering workflow, by establishing the ideal position and morphology of the planned restoration first followed by the virtual planning and placement of the dental implant(s). ${ }^{2}$ Two types of GIS protocols, static and dynamic, are described in the literature. The static approach, better known as computer-guided surgery or GIS. ${ }^{3}$ The static navigation can further be divided into full- and half-GIS. The half-guided implant placement includes the drillingguided, pilot-drill-guided, and non-computer-guided approaches. ${ }^{4}$ Dynamic-guided surgery, also called navigation, reproduces the virtual implant position directly from computerized tomographic data and uses motion-tracking technology to guide the implant osteotomy preparation. ${ }^{3,4}$ Non-computer GIS procedures require more surgical experience to overcome their limitations.

Despite more than a decade of clinical and research pieces of evidence, and with improved technologies in instrumentation and techniques, the use of GIS remains subject to deviations between the planned and achieved implant positions. ${ }^{5}$ As GIS typically includes intraoral cone-beam computed tomography (CBCT) with or without intraoral scanning (IOS) followed by the superimposition of the CBCT and IOS and virtual placement of restorations and implants with drill-guides and with or without anchor-pins. Furthermore, a whole virtual 3D planning can be either 3D printed (additive) or milled (subtractive) into the surgical template that can be used with a system-specific GIS kit. Substantial accumulative errors can occur at each of these individual steps, resulting in potentially disastrous deviations from proper implant placement. ${ }^{6}$ Orentlicher et al. ${ }^{1}$ nicely summarized the myths of GIS and provided scientifically evidenced answers to each myth referring to their accuracies, indications, time-requirement, ease of treatment, insertion torques, bone overheating, and cumulative survival rate.

These problems can be classified into the following categories:

- Software-based errors: These are inherent to each system and the accuracy is based on each software's ability to convert digital information into interactive planning information. Few companies have closed-systems from start to end and those offering open-system may have to depend upon the compatibility of digital data acquisition, manipulation, and reproduction. The errors may be incorporated during any of these data transfer stages.

- Hardware-based errors: These are the errors based on the accuracies of materials and types of equipment and techniques used for 3D printing of the surgical templates. Each material and
Division of Clinical Dentistry (Prosthodontics), School of Dentistry, International Medical University, Kuala Lumpur, Malaysia

Corresponding Author: Pravinkumar G Patil, Division of Clinical Dentistry (Prosthodontics), School of Dentistry, International Medical University, Kuala Lumpur, Malaysia, Phone: +601135022042 , e-mail: pravinandsmita@yahoo.co.in

How to cite this article: Patil PG. Problems of Guided Implant Surgery. Int J Prosthodont Restor Dent 2020;10(4):138-139.

Source of support: Nil

Conflict of interest: None

a technique (whether it is additive or subtractive) have their inherent margins of errors.

- Affordability and accessibility of software and hardware support: Sometimes clinicians must depend upon the industrial partners to get the software designing support or 3D printing or milling support. All-inclusive of additional expenses and may not be affordable to all the patients. Limited accessibility and availability of specific digital service providers due to geographical location could be another potential problem causing a delay in the treatment.

- The complexity of arch anatomy, form, and shape:These are local intraoral problems pertaining to anatomical ridge-shape and -form. No arch is perfect for implants and frequently needs simple to complex modifications before implant placement with or without bone grafting. If the ridge needs complex modifications before surgery, the errors may be incorporated at these stages even for fully-guided protocols. Completely edentulous arches are the most complex and difficult for attempting GIS procedures as they frequently need additional anchor-pins for template stabilization. Resorbed ridges may not always provide a space for such anchor-pins placement and surgeons must depend upon alternate means of stabilization.

- Patient intraoral limitations: Limited mouth opening is one of the most limiting factors to allow the surgical drills to pass through the template guide hole, especially in the posterior region, and the clinician has no choice but to depend upon their own expertise without any template. Microstomia and oral submucous fibrosis are few clinical conditions that compromise the patients' mouth opening range as well.

- Surgical procedural limitations: Implant placement includes the use of surgical handpieces with internal or external irrigation as a coolant to minimize heat generation in the bone. The surgical template remains a barrier between the drills and the source of external irrigation causing a less colling effect. If the surgery is flapless (in many fully guided procedures) then the mucosa also remains another barrier. The research has shown higher heat generation in the bone during implant placement

(c) Jaypee Brothers Medical Publishers. 2020 Open Access This article is distributed under the terms of the Creative Commons Attribution 4.0 International License (https://creativecommons.org/licenses/by-nc/4.0/), which permits unrestricted use, distribution, and non-commercial reproduction in any medium, provided you give appropriate credit to the original author(s) and the source, provide a link to the Creative Commons license, and indicate if changes were made. The Creative Commons Public Domain Dedication waiver (http://creativecommons.org/publicdomain/zero/1.0/) applies to the data made available in this article, unless otherwise stated. 
with surgical templates compared with implant placement without the templates, however, within clinically acceptable limits. The use of internal irrigation drills minimizes such problems to a greater extent.

- Level of competency and knowledge of a clinician: The level of competency of the clinician in handling the GIS procedure is a crucial factor especially in completely edentulous arches. The specific learning curve must be practiced by all clinicians by completing the recognized implant programs and trusted continues professional-developmental courses. Detailed knowledge of the GIS systems and tools is a crucial factor in successful implant placement.

- Human errors: These are inherent errors that may occur at any stage of the process including those who are performing IOS, those who are doing software planning, and those who are performing the surgery. No matter how accurate the printed template is, if the surgeon does even a minor error in intraoral placement or failed to detect the misfit of it, the implant position may go in a disastrously wrong way. The clinician's tactile sensation toward judging the bone density becomes compromised while using GIS procedures and can add another potential error in achieving clinically acceptable implant stability and position accuracy. ${ }^{7}$ Consistent verification of both diagnostic and surgical procedures after each step can minimize these errors.

Despite these problems, the GIS has been refined for many years and can be used in most clinical scenarios. Attention to detail is necessary for all steps to minimize potential cumulative errors. Cunha et al. ${ }^{5}$ analyzed a total of 61 implants for their virtually planned position and actual placed position of the implants and found out the mean angular deviation of about 2.04 degrees with no statistically significant difference between the planned and placed position of the implants. ${ }^{5}$ In a 7-year retrospective study of 798 implants placed fully guided using different software and implant systems, the authors reported a cumulative survival rate of $96.98 \%$ compared with the well-established cumulative survival rate of 95-98\% for conventional freehand placement of implants. ${ }^{8}$

Unfortunately, the problems or errors with GIS have not been sufficiently highlighted in the literature. More clinical and laboratory research should be focused on identifying and correcting these problems.

\section{References}

1. Orentlicher G, Horowitz A, Goldwaser B, et al. Ten myths of guided implant surgery. Compend Contin Educ Dent 2017;38(8):552-557.

2. Orentlicher G, Horowitz A, Abboud M. Minimally invasive implant surgery using computer-guided technology. In: Minimally Invasive Dental Implant Surgery Cullum D, Deporter D, ed., Hoboken, NJ: Wiley Publications; 2016. pp. 169-189.

3. D'haese J, Ackhurst J, Wismeijer D, et al. Current state of the art of computer-guided implant surgery. Periodontol 2000 2017;73(1):121133. DOI: $10.1111 /$ prd.12175.

4. Gargallo-Albiol J, Barootchi S, Salomó-Coll O, et al. Advantages and disadvantages of implant navigation surgery. A systematic review. Ann Anat 2019;225:1-10. DOI: 10.1016/j.aanat.2019.04.005.

5. Cunha RM, Souza FÁ, Hadad H, et al. Accuracy evaluation of computer-guided implant surgery associated with prototyped surgical guides. J Prosthet Dent 2021;125(2):266-272. DOI: 10.1016/j. prosdent.2019.07.010.

6. Tatakis DN, Chien HH, Parashis AO. Guided implant surgery risks and their prevention. Periodontol 2000 2019;81(1):194-208. DOI: 10.1111/ prd.12292.

7. Patil PG. Clinician's tactile sensation and dental implantology. Int J Prosthodont Restor Dent 2013;3(3):vi.

8. Orentlicher $G$, Horowitz A, Goldsmith D, et al. Cumulative survival rate of implants placed "fully guided" using CT-guided surgery: a 7-year retrospective study. Compend Contin Educ Dent 2014;35(8):590-598, 600. 